\title{
The Malawi trauma score: A model for predicting trauma-associated mortality in a resource-poor setting $\star$
}

\author{
Jared Gallaher $^{\mathrm{a}}$, Malcolm Jefferson ${ }^{\mathrm{a}}$, Carlos Varela ${ }^{\mathrm{b}}$, Rebecca Maine ${ }^{\mathrm{a}}$, Bruce Cairns ${ }^{\mathrm{a}, \mathrm{b}, \mathrm{c}}$, \\ Anthony Charles $\mathrm{s}^{\mathrm{a}, \mathrm{b}, \mathrm{c}, *}$ \\ a Department of Surgery, University of North Carolina School of Medicine, Chapel Hill, NC, USA \\ ${ }^{b}$ Department of Surgery, Kamuzu Central Hospital, Lilongwe, Malawi \\ ${ }^{\mathrm{c}}$ North Carolina Jaycee Burn Center, Department of Surgery, University of North Carolina School of Medicine, CB\# 7600, Chapel Hill, NC, USA
}

A R T I C L E I N F O

Keywords:

Global health

Trauma

Injury

Injury severity scoring

\begin{abstract}
A B S T R A C T
Background: Globally, traumatic injury is a leading cause of morbidity and mortality in low-income countries. Current tools for predicting trauma-associated mortality are often not applicable in lowresource environments due to a lack of diagnostic adjuncts. This study sought to derive and validate a model for predicting mortality that requires only a history and physical exam.

Methods: We conducted a retrospective analysis of all patients recorded in the Kamuzu Central Hospital trauma surveillance registry in Lilongwe, Malawi from 2011 through 2014. Using statistical randomization, $80 \%$ of patients were used for derivation and $20 \%$ were used for validation. Logistic regression modeling was used to derive factors associated with mortality and the Malawi Trauma Score (MTS) was constructed. The model fitness was tested.

Results: 62,354 patients are included. Patients are young (mean age 23.0, SD 15.9 years) with a male preponderance (72\%). Overall mortality is $1.8 \%$. The MTS is tabulated based on initial mental status (alert, responds to voice, responds only to pain or worse), anatomical location of the most severe injury, the presence or absence of a radial pulse on examination, age, and sex. The score range is 2-32. A mental status exam of only responding to pain or worse, head injury, the absence of a radial pulse, extremes of age, and male sex all conferred a higher probability of mortality. The ROC area under the curve for the derivation cohort and validation cohort were 0.83 (95\% CI 0.78, 0.87) and 0.83 (95\% CI 0.75, 0.92), respectively. A MTS of 25 confers a $50 \%$ probability of death.

Conclusions: The MTS provides a reliable tool for trauma triage in sub-Saharan Africa and helps risk stratify patient populations. Unlike other models previously developed, its strength is its utility in virtually any environment, while reliably predicting injury- associated mortality.
\end{abstract}

\section{Background}

The global burden of traumatic injury and its disproportionate effect on low- and middle-income countries (LMICs) is well described. [1,2] While traumatic injury accounts for $16 \%$ of the world's adult morbidity and premature death, LMICs suffer $90 \%$ of the associated mortality [3-5]. Consequently, public health efforts

\footnotetext{
$\star$ These data were presented as an oral presentation at the National Committee on Trauma National Paper Competition in San Antonio, TX on March 8, 2018 and was the Clinical Paper Second Place Winner. These data were also presented as an oral presentation at the Academic Surgical Congress Annual Conference in Jacksonville, FL on February 1, 2018.

* Corresponding author at: School of Medicine, University of North Carolina, 4008 Burnett Womack Building, CB 7228, USA.

E-mail address: anthchar@med.unc.edu (A. Charles).
}

over the last decade have increasingly recognized the importance of a national health policy that strengthens regional and national trauma systems as part of a balanced effort to improve health care in low-resource regions such as sub-Saharan Africa. However, good health care policy can only be informed by good data, not only on overall health system capacity, but also on regional patterns and burden of trauma, including patient volume and injury severity. Trauma surveillance registries are an essential first step of improving trauma care in resource-poor settings [6].

To accurately describe the burden of trauma, injury severity must be characterized in a manner that allows for objective comparisons between patient populations. This is essential for the risk stratified characterization of observed and expected trauma outcomes in a given population. Injury scoring systems are also useful in trauma triage, especially in austere environments, where constant prioritization in health care resource 
allocation is necessary and will help clinicians identify patients most at risk of death.

The Injury Severity Score (ISS) was developed for this purpose nearly fifty years ago and has been ubiquitous in its use in the United States and Europe since its introduction [7]. It is a graded, system-based score that requires detailed injury information based on diagnostic imaging adjuncts. The Revised Trauma Score (RTS) was published several years later as an alternative scoring system with much simpler clinical variables, only requiring the patient's initial Glasgow Coma Scale (GCS), systolic blood pressure, and respiratory rate [8]. Other classification schemes such as the Trauma-Injury Severity Score (TRISS), the New Injury Severity Score (NISS), the ICD-9 Injury Severity Score (ICISS), and A Severity Characterization of Trauma (ASCOT) have iterated on these original ideas but with similar data requirements and performance at predicting post-injury mortality [9-12].

The utility of these scoring systems in a resource-poor environment is somewhat limited. The Injury Severity Score and its other proxies require the acquisition of granular data on each injury from predefined body regions. These scoring systems require advanced imaging such as computerized tomography (CT) scans, which, though ubiquitous in high-income countries (HICs), are rarely available in low-resource countries. In addition, the ISS is a retrospective scoring system, giving it limited utility for trauma triage or transfer decision-making. To address these challenges, the Kampala Trauma Score (KTS) was developed in Uganda [13]. However, it has not been updated in almost twenty years and we have previously shown that it has limited utility in our trauma patient population [14].

At our tertiary center in Lilongwe, Malawi, we sought to describe injury severity in an accurate and reproducible manner by developing a scoring system that would require only a history and physical exam, while also predicting trauma-associated mortality. This score would have potential as a foundation for rapid triage tools at regional and tertiary trauma centers throughout the region and allow for risk-stratification of our patient populations.

\section{Methods}

This study is a retrospective analysis of data from the Kamuzu Central Hospital (KCH) Trauma Registry. $\mathrm{KCH}$ is a public 600-bed tertiary care hospital in the capital city of Lilongwe, which serves as a referral center for approximately 6 million people in the central region of Malawi. $\mathrm{KCH}$ is equipped with six intensive care unit (ICU) beds and six ventilators and a surgical step-down unit. Trauma and orthopedic surgical services are available seven days a week. Surgical consultants, Malawian general surgery and orthopedic registrars, and clinical officers staff the trauma service.

The KCH Trauma Registry was established in 2008 in conjunction with the Malawi Ministry of Health to collect patient demographic information, clinical characteristics, and outcome data of all patients presenting to the emergency department with traumatic injuries. Research staff are available in the emergency department twenty-hours a day, seven days a week, to collect and record data. We have previously demonstrated how trauma registry clerks are trained in the acquisition of data, such as vital signs [15]. Specifically, data points utilized in this study include age, sex, date of injury, setting of injury, mechanism of injury, type and location of injury, clinical scoring systems such as the Alert, Voice, Pain, Unresponsive (AVPU) Scale, systolic blood pressure, and outcome (discharge or death). The AVPU scale records a patient's level of consciousness as either alert, responding to verbal stimuli, responding to pain stimuli, or unresponsive. It correlates with GCS and is simple to calculate [16]. The presence of a radial pulse on exam was defined as being equivalent to a systolic blood pressure of greater than $90 \mathrm{mmHg}$ [17]. Primary injury location was defined as the anatomic location of the most severe injury as diagnosed by the trauma care provider. For example, for soft tissue injuries, a laceration would be more severe than a bruise, and violation of body cavity would be more severe than a laceration. Or as another example, an open fracture would be more severe than a closed fracture and head trauma with loss of consciousness would take precedence over a soft tissue injury. All patients who presented to the emergency department with traumatic injuries over four years between January 2011 and December 2014 were included in this study. Baseline characteristics, with standard deviations when applicable, are described. The primary outcome measure used was crude in-hospital mortality compared to survival at time of discharge from the hospital.

To evaluate for the predictors of trauma mortality within the clinical variables in the dataset, the study population was partitioned into two cohorts. A simple random sample (without replacement) with a selection probability of 0.8 was drawn from the original set of patients and designated as the sample from which the scoring function would be derived (derivation cohort). The remaining cases were used for internal validation of the newly constructed score (validation cohort). Only patients with a complete record of all included variables were included in the model.

The sum of the derived odds ratios from the variables included in the logistic regression model were then used to construct the Malawi Trauma Score (MTS). Logistic regression modeling was utilized to evaluate the performance of the MTS and predict patient survival probabilities within the derivation and validation cohort. Forward stepwise selection was performed to evaluate a variable's association with mortality in the derivation set. The Hosmer and Lemeshow (H-L) goodness-of-fit test was used to determine each model's adequacy for these data. This test places subjects into deciles based on the model-predicted probabilities, then computes a Pearson chi-square test based on the observed and expected number of subjects in the deciles [18]. This strategy was deemed particularly appropriate for measuring goodness of fit of our models which utilize qualitative and categorical explanatory variables. Our results were similar when compared with a modified H-L methodology [19]. Receiver operating characteristic (ROC) analyses were performed to evaluate the performance of the MTS.

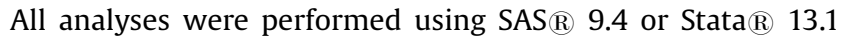
software. The University of North Carolina Institutional Review Board and the Malawi National Health Services Review Committee approved this study.

\section{Results}

During the study period, 62,354 patients with traumatic injuries presented to $\mathrm{KCH}$. Patients were predominantly male (72.0\%) and relatively young with a mean age of 23.0 (SD 15.9 years). (Table 1) Patients predominantly presented after a fall (29.5\%). Motor vehicle related trauma accounted for $25.0 \%$ of the injuries, with $17.0 \%$ of patients as a driver or a passenger in a motor vehicle collision and $8.0 \%$ struck by a vehicle. Assault was the third most common mechanism at $24.0 \%$ while the remaining injury mechanisms were relatively rare. Almost half of patients, $43.5 \%$, were transported to the hospital via minibus, a small passenger van working as a taxi. A third of patients, $33.2 \%$, arrived via private vehicle, and only $10.9 \%$ were transported by an ambulance.

The most common injury location was head or face at 18,120 patients (29.3\%) followed by lower extremities at 14,391 patients (23.1\%) and upper extremities at 12,256 patients (19.7\%). (Table 1) Hand injuries were also frequent with 7632 patients (12.2\%). Injuries of the spine, chest, abdomen/flank, and pelvis all comprised less than five percent each of the overall injury pattern. Most patients had normal initial vital signs with a mean heart rate 
Table 1

Background information on patients presenting with traumatic injury.

All patients

$(n=62,354)$

Patient Age (years)

Mean (SD)

Gender: N (\%)

Female

Male

Missing

Mechanism of injury: N (\%)

Pedestrian hit by vehicle

Driver/passenger in vehicle accident

Fall

Assault

Collapsed Structure

Other

Missing

Transport to Hospital

Minibus

Private Vehicle

Ambulance

Walked

Police

Other

Missing

Injury Type: $\mathbf{N}(\%)$

Soft Tissue Injury

Fracture

Dislocation

Head Injury

Penetrating Wound

Burn

Other

Missing

Injury Location: N (\%)

Head/face

Spine

Upper Extremity

Hand

Chest

Abdomen/Flank

Pelvis

Lower Extremity

Missing

Initial Heart Rate

Mean (SD)

Initial SBP (mean)

Mean (SD)

Initial AVPU

Alert

Responds to Voice

Responds to Pain

Unresponsive

Missing

Mortality: N (\%)

Overall crude mortality
23.0 (15.9)

$17,460(28.0)$

44,869 (72.0)

$25(0.0)$

4,981 (8.0)

10,617 (17.0)

$18,421(29.5)$

14,963 (24.0)

3,184 (5.1)

$9,879(15.8)$

309 (0.5)

27,138 (43.5)

20,674 (33.2)

6,788 (10.9)

2,621 (4.2)

2,416 (3.9)

$2,550(4.1)$

$167(0.3)$

$39,950(64.1)$

9,952 (16.0)

2,532 (4.1)

2,407 (3.9)

1,196 (1.9)

2,792 (4.5)

$3,373(5.4)$

$152(0.2)$

$18,120(29.3)$

2,994 (4.8)

12,256 (19.7)

7,632 (12.2)

3,094 (5.0)

2,116 (3.4)

1,336 (2.1)

14,391 (23.1)

427 (0.7)

86 bpm (18)

$125 \mathrm{mmHg}$ (17)

61,166 (98.1)

$188(0.3)$

$104(0.2)$

639 (1.0)

$527(0.4)$

$1,120(1.8)$

of 86 (SD $18 \mathrm{bpm}$ ), and a mean systolic blood pressure of 124 (SD $17 \mathrm{mmHg}$ ). Based on the AVPU scale, 61,166 patients (98.1\%) were alert and 188 patients $(0.3 \%)$ were responsive to voice. Only 639 patients (1.0\%) were unresponsive. Among all patients, crude mortality was $1.8 \%$.

The Malawi Trauma Score (MTS) is comprised of five components: AVPU score, anatomic location of injury, the presence or absence of a radial pulse on exam, age, and sex. (Table 2) The score ranges from a minimum of 2 to a maximum of 32. An AVPU score corresponding with responsiveness to pain or unresponsiveness confers a score of 10 points, while responsiveness to voice scores one point. A fully alert patient is given zero points. Patients at the extreme of ages for our trauma population (0-9 years and $>$ 33 years old) are given 4 points. Those aged $10-23$ years are given 1 point and those $24-33$ years old are given 3 points. Female sex confers no points and male sex, 3 points.

Table 2

The Malawi Trauma Score (MTS). It ranges from 2 to 32 points.

\begin{tabular}{lll} 
& Value & $\begin{array}{l}\text { +Points of } \\
\text { the MTS }\end{array}$ \\
AVPU & 4 & 0 \\
& 3 & 1 \\
Age & 2 or 1 & 10 \\
& $10-23$ & 1 \\
Sex & $24-33$ & 3 \\
& $0-9$ or $>33$ & 4 \\
Radial Pulse & Female & 0 \\
\multirow{3}{*}{ Injury Location } & Male & 3 \\
& Radial pulse present & 0 \\
& Radial pulse absent & 10 \\
& Pelvis/Lower Extremity & 1 \\
& Hand/Chest/Upper Extremity & 2 \\
Abdomen/Flank & 3 \\
& Head/Face/Spine & 5
\end{tabular}

The presence of a radial pulse on exam adds no points but the absence of a radial pulse confers 10 points. Anatomic location of injury location is the final component. Injuries to the pelvis or lower extremity add 1 point, with injuries to the hand, chest, or upper extremity add 2 points. Abdominal or flank injuries confer 3 points with injures to the head, face, or spine confers 5 points.

Our model only included patients with complete data for the utilized variables. The randomly generated derivation set included 21,417 patients while the randomly generated validation model included 5412 patients. The initial model, from which the odds ratios were derived to create MTS, was found to be adequate for the data with a H-L statistic of $6.07(p=0.64)$. MTS was also found to be an adequate fit for the data of the derivation cohort with an $\mathrm{H}-\mathrm{L}$ Statistic of $6.394(p=0.60)$. Additionally, AUC analysis of the ROC curves resulting from the model (with original predictors) and MTS (applied to the derivation set) was shown not to be statistically different $(p=0.78$ ). This suggests that no significant information is lost in the transformation of the model predictors into a single, simplified trauma score. Validation of the score also revealed that MTS performs as well as the original model in predicting mortality in trauma patients, with the model producing an adequate fit to the validation data set ( $\mathrm{H}-\mathrm{L}$ statistic: $10.34, p=0.32$ ). ROC analyses showed the derivation cohort to have an adequate discrimination with an area under the curve (AUC) of 0.83 (95\% CI $0.78,0.87$ ).

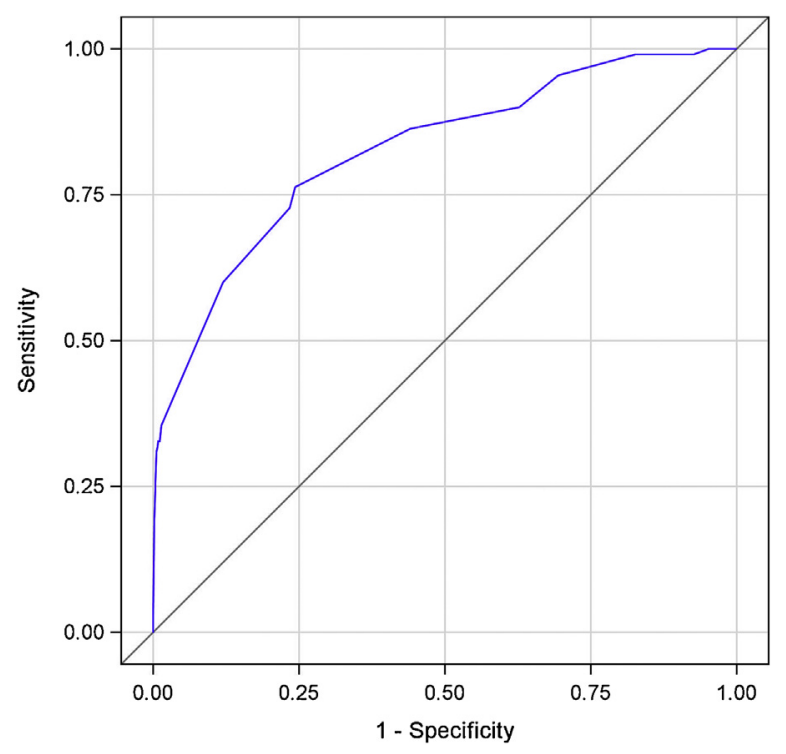

Fig. 1. ROC Curve for the derivation cohort, $A U C=0.83$ (95\% CI $0.78,0.87$ ). 
(Fig. 1) The internal validation cohort was equivalent with an AUC of 0.83 (95\% CI 0.75, 0.92). (Fig. 2)

The observed mortality increased as the MTS increased as shown in Fig. 3. This association was strongest as the MTS approached a score of 20 . The association with mortality when fit to a logistic regression model is also shown overlying the raw data with its 95\% confidence interval. In Table 3, we report the observed versus predicted mortality at each level of MTS observed in the validation cohort. At a score of approximately 25 , the predicted probability of death was nearly $50 \%$. At a score of 30 , near the maximum of 32 , the probability of death was close to $80 \%$.

The systolic blood pressure or palpable radial pulse was not recorded in 33,468 patients (53.7\%) but sensitivity analysis suggests that patients with and without this data had a relatively similar severity of injury. AVPU scores were comparable between the two groups with a few more patients scoring a 2 or 1 in those without a recorded SBP or radial pulse. These patients also scored more points in the MTS for extreme age. (Table 4). However, there were fewer males in the missing group, $75.9 \%$ versus $68.6 \%$ ( $\mathrm{p}<0.001$ ), and they had lower injury location scores, especially in head/face/spine injuries with $39.1 \%$ in the recorded group and $29.3 \%$ in the missing group $(p<0.001)$. When excluding the missing radial pulse component, the total MTS score of the remaining four components was the same between the two groups at 8.0 (SD 2.6) in the group with a recorded systolic blood pressure or palpable radial pulse and 8.0 (SD 2.9) in the group missing it $(\mathrm{p}=0.1)$. Age was missing in 174 patients $(0.3 \%)$, sex in 17 patients $(0.03 \%)$, AVPU in 257 patients $(0.4 \%)$, and anatomic injury location in 362 patients $(0.6 \%)$. Other utilized data points were missing in less than two percent of patients for all remaining variables.

\section{Discussion}

The Malawi Trauma Score is a reliable tool for assessing injury severity in sub-Saharan Africa. Its strength is its utility in virtually any environment, while reliably predicting injury-associated mortality. The MTS may also have value as an instrument for trauma triage and for risk-stratifying populations.

The MTS is one of many tools emerging from LMICs developed to address the resource constraints associated with trauma care delivery $[20,21]$. It is challenging to assess injury severity in a lowresourced setting where advanced diagnostic adjuncts are

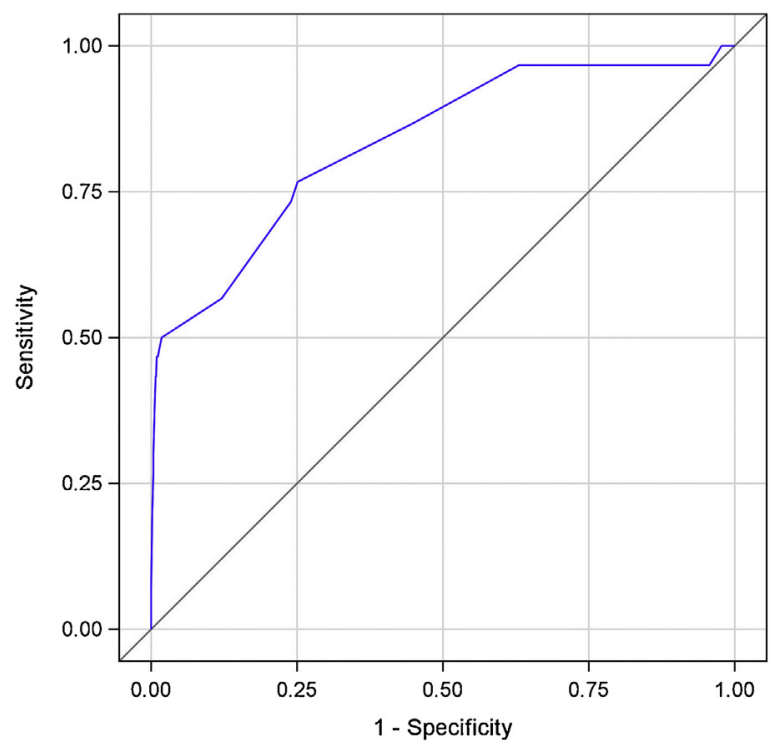

Fig. 2. ROC Curve for the internal validation cohort, $A U C=0.83(95 \% \mathrm{CI} 0.75,0.92)$.

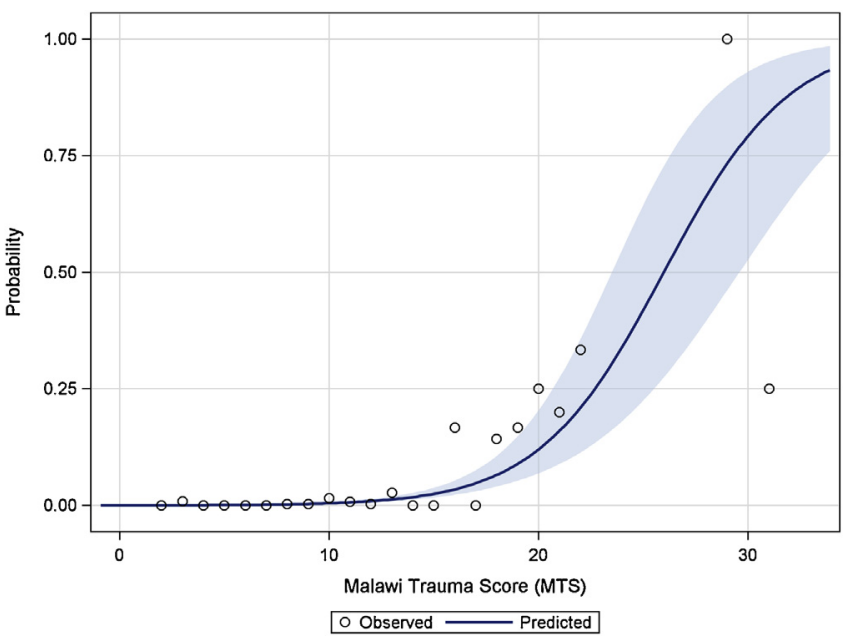

Fig. 3. Crude mortality of patients in the validation cohort by MTS score plotted against the predicted probability of death based on MTS score with $95 \% \mathrm{CI}$.

expensive to obtain and even more difficult to maintain. In Malawi, district hospitals are especially understaffed with minimal capacity for complex trauma management [22]. The MTS may be most valuable at these facilities where it can serve as a guide for rapid triage and transfer to a tertiary center.

The Kampala Trauma Score (KTS) was developed to address the limitations of conventional scoring systems in environments throughout sub-Saharan Africa [13]. It included five clinical components: age, systolic blood pressure, respiratory rate, AVPU, and the number of serious injuries, as assessed by the clinician. While it has been widely used for over a decade, data on the accuracy and utility of KTS are both limited and inconsistent. Two recent studies on the utility of KTS from West Africa have shown favorable comparisons to other scoring systems such as the RTS and the ISS in predicting mortality [23,24]. A 2017 study from Kenya showed adequate performance across several hospitals but other data from East Africa, India, and South America have been mixed [14,25-28]. At our center, we compared the RTS and the KTS on a cohort of approximately 16,000 trauma patients. Both scoring systems were comparable as predictors of mortality but neither had satisfactory discrimination, especially for admitted trauma patients [14].

The MTS offers several advantages. First, the MTS is simple and practical to record. It does not require any diagnostic tools, even a blood pressure cuff despite a potential limitation that the MTS requires a subjective provider assessment of a the most severe injury when assigning injury location data, especially in complex multi-system trauma. Simplicity is vital for maintaining an accurate and complete trauma registry, especially at high trauma volume centers in resource-poor settings. In addition, the MTS provides a useful and clear cutoff for severe versus non-severe injuries for use in trauma triage. Predicted mortality clearly increases exponentially at a score of 18 , which can serve as marker for regional transfer to a tertiary center or in triage decisions at more advanced facilities. Lastly, the MTS was developed on a much larger cohort of patients with more recent data and was internally validated. Previous scoring systems were largely based on small number of patients, and KTS is now almost twenty years old. Experience shows that conditions continue to evolve rapidly throughout this region and that our assessment of available resources and clinical outcomes needs constant revalidation. These changes, and the heterogeneity of national trauma systems in subSaharan Africa, may explain the variable results of the KTS in predicting mortality across different populations. 
Table 3

Observed versus predicted mortality at each observed level of MTS for the validation cohort.

$\begin{array}{lllll}\text { MTS } & \text { Patients }(\mathrm{n}) & \text { Deaths }(\mathrm{n}) & \begin{array}{l}\text { Observed } \\ \text { Mortality }(\%)\end{array} & \begin{array}{l}\text { Predicted } \\ \text { Mortality (\%) }\end{array} \\ 2 & & & 0.00 & 0.03 \\ 3 & 121 & 0 & 0.87 & 0.05 \\ 4 & 115 & 1 & 0.00 & 0.07 \\ 5 & 130 & 0 & 0.00 & 0.09 \\ 6 & 532 & 0 & 0.00 & 0.13 \\ 7 & 737 & 0 & 0.00 & 0.18 \\ 8 & 354 & 0 & 0.30 & 0.25 \\ 9 & 985 & 3 & 0.28 & 0.35 \\ 10 & 1064 & 3 & 1.61 & 0.48 \\ 11 & 62 & 1 & 0.78 & 0.67 \\ 12 & 643 & 5 & 0.36 & 0.94 \\ 13 & 557 & 2 & 2.70 & 1.30 \\ 14 & 37 & 1 & 0.00 & 1.81 \\ 15 & 2 & 0 & 0.00 & 2.51 \\ 16 & 6 & 0 & 16.67 & 3.47 \\ 17 & 6 & 1 & 0.00 & 4.78 \\ 18 & 4 & 0 & 14.29 & 6.54 \\ 19 & 14 & 2 & 16.67 & 8.90 \\ 20 & 12 & 2 & 25.00 & 12.00 \\ 21 & 10 & 1 & 20.00 & 15.99 \\ 22 & 12 & 2 & 33.33 & 20.99 \\ 29 & 1 & 4 & 100.00 & 73.26 \\ 31 & 4 & 1 & 25.00 & 84.22 \\ & & 1 & & \end{array}$

Table 4

Components of the MTS stratified by patients with and without a recorded palpable radial pulse or systolic blood pressure.

$\begin{array}{lll}\begin{array}{l}\text { Points } \\ \text { of the }\end{array} & \begin{array}{l}\text { Radial Pulse or } \\ \text { SBP Recorded }\end{array} & \begin{array}{l}\text { Radial Pulse or } \\ \text { SBP Not }\end{array} \\ \text { MTS } & (n=28,886) & \begin{array}{l}\text { Recorded } \\ (n=33,468)\end{array}\end{array}$

\begin{tabular}{|c|c|c|c|c|}
\hline \multicolumn{5}{|l|}{ AVPU: N (\%) } \\
\hline 4 & 0 & $28,579(98.9)$ & 32,418 (96.9) & \multirow[t]{4}{*}{$<0.001$} \\
\hline 3 & 1 & $7(0.1)$ & $181(0.5)$ & \\
\hline 2 or 1 & 10 & $89(0.3)$ & $654(2.0)$ & \\
\hline Missing & - & $42(0.2)$ & $215(0.6)$ & \\
\hline \multicolumn{5}{|l|}{ Age: $\mathbf{N}(\%)$} \\
\hline $10-23$ & 1 & 7,989 (27.7) & $8,015(24.0)$ & \multirow[t]{4}{*}{$<0.001$} \\
\hline $24-33$ & 3 & $11,252(39.0)$ & $5,139(15.4)$ & \\
\hline $0-9$ or $>33$ & 4 & $9,603(33.2)$ & $20,117(60.1)$ & \\
\hline Missing & - & $42(0.2)$ & $197(0.6)$ & \\
\hline \multicolumn{5}{|l|}{ Sex: $N(\%)$} \\
\hline Female & 0 & $6,964(24.1)$ & $10,496(31.4)$ & \multirow[t]{3}{*}{$<0.001$} \\
\hline Male & 3 & $21,920(75.9)$ & $22,949(68.6)$ & \\
\hline Missing & - & $2(0.0)$ & $23(0.1)$ & \\
\hline \multicolumn{5}{|l|}{ Injury Location: N (\%) } \\
\hline Pelvis/Lower Extremity & 1 & $7,023(24.3)$ & $8,704(26.0)$ & \multirow[t]{2}{*}{$<0.001$} \\
\hline $\begin{array}{l}\text { Hand/Chest/Upper } \\
\text { Extremity }\end{array}$ & 2 & $9,764(33.8)$ & $13,218(39.5)$ & \\
\hline Abdomen/Flank & 3 & $698(2.4)$ & $1,418(4.2)$ & \\
\hline Head/Face/Spine & 5 & $11,293(39.1)$ & $9,809(29.3)$ & \\
\hline Missing & - & $108(0.4)$ & $319(1.0)$ & \\
\hline $\begin{array}{l}\text { MTS Score Excluding } \\
\text { Missing Radial Pulse } \\
\text { Component: } \\
\text { Mean (SD) }\end{array}$ & & $8.0(2.6)$ & $8.0(2.9)$ & 0.1 \\
\hline
\end{tabular}

The local development of a trauma score raises important questions about its applicability in other contexts. This makes it important to validate its use in other regions and other patient populations. We acknowledge that the major limitation of the MTS is its derivation based on a specific regional population with particular injury and demographic patterns. However, previous data shows that our population is comparable to others throughout sub-Saharan Africa, increasing the likelihood that our model will have utility outside Malawi [29,30]. An important next step is validating the MTS's utility in another tertiary center or regional trauma center in sub-Saharan Africa.
Evidence also suggests that the MTS may be useful in highincome countries. Two recent studies found the KTS to be at least equal, if not better, at predicting mortality compared to conventional scoring systems such as the ISS and TRISS when using data from US trauma databases [31,32]. Additionally, Akay et al showed that while TRISS was superior, the KTS still performed well at predicting mortality [33]. Given these findings, it is likely that the MTS would also perform well in these environments. The use of the MTS in a high-income country setting has potential to simplify data collection and improve triage, especially in rural trauma centers or in the pre-hospital setting. In contrast to the ISS, the MTS can be calculated in the field and in real-time. Prior data have demonstrated that pre-hospital scoring systems have potential benefit for triage, but there is not a consensus on a particular system $[34,35]$. Like the ISS, many of these tools are relatively complex compared to the MTS, even those developed in LMICs [36]. The MTS's potential as a triage tool needs further investigation both in the pre-hospital setting and in rural emergency departments in the US.

Lastly, our study highlights the complex challenges of capturing trauma-related outcomes in a resource-poor environment. Maintaining a trauma registry with both accuracy and completeness is very challenging in high volume centers where a robust health care infrastructure is lacking. Previous data from similar settings suggests that many trauma centers are collecting the information needed to calculate the MTS, but experience in the region demonstrates that improvements in both the quality and quantity of data will be gradual [37]. Despite the limitations of missing data in our registry, our complete model represents one of the largest cohorts of trauma patients reported in sub-Saharan Africa. Sensitivity analysis of our missing data suggests that this data was missing at random and that our complete set is representative of our trauma population as a whole.

\section{Conclusion}

The Malawi Trauma Score is a simple, but reliable predictor of trauma-associated mortality. Its advantage is its reliance only on a history and physical examination. This makes it applicable in both low and high-resource environments as well as the prehospital and emergency department setting. Further work is needed to validate the MTS in other regions in sub-Saharan Africa and to explore its utility in high-income countries.

\section{Author's contribution}

Jared R. Gallaher, MD, MPH contributed to this paper by: design of study, acquisition, analysis, and interpretation of data, drafting and revision of the manuscript, and statistical analysis.

Carlos Varela MB,BS contributed to this paper by: design of study, critical revision of the manuscript for important intellectual content, and administrative and technical support.

Rebecca Maine MD, MPH contributed to this paper: interpretation of data, critical drafting and revision of the manuscript, and statistical analysis

Malcolm Jefferson, MD, MS contributed to this paper by: design of study, statistical analysis, and critical revision of the manuscript for important intellectual content

Bruce A. Cairns, MD contributed to this paper by: design of the study, revision of the manuscript for important intellectual content, supervision, and the obtaining of funding.

Anthony G. Charles, MD, MPH contributed to this paper by: conception and design of the study, acquisition, analysis, and interpretation of data, drafting and revision of the manuscript, statistical analysis, obtaining of funding, and supervision. 


\section{Acknowledgements}

Financial support was provided by the North Carolina Jaycee Burn Center in the Department of Surgery at the University of North Carolina for all aspects of the study including: design and conduct of the study; collection, management, analysis, and interpretation of the data; and preparation, review, or approval of the manuscript.

\section{References}

[1] Gallaher JR, Haac BE, Geyer AJ, Mabedi C, Cairns BA, Charles AG. Injury characteristics and outcomes in elderly trauma patients in Sub-Saharan Africa. World J Surg 2016;40(November (11)):2650-7.

[2] Samuel JC, Akinkuotu A, Villaveces A, Charles AG, Lee CN, Hoffman IF, et al. Epidemiology of injuries at a tertiary care center in Malawi. World J Surg 2009;33(September (9)):1836-41.

[3] Mathers CD, Lopez AD, Murray CJL. The burden of disease and mortality by condition: data, methods, and results for 2001. In: Lopez AD, editor. Global burden of disease and risk factors. New York City, NY: Oxford University Press; 2006.

[4] World Health Organization. Estimates for 2000-2012: cause-specific mortality. 2012. . Accessed November 03, 2018 http://www.who.int/ healthinfo/global_burden_disease/estimates_regional_2000_2012/en/.

[5] Abubakar II, Tillmann T, Banerjee A. Global, regional, and national age-sex specific all-cause and cause-specific mortality for 240 causes of death, 19902013: a systematic analysis for the Global Burden of Disease Study 2013. Lancet 2015;385(January (9963)):117-71.

[6] Nwomeh BC, Lowell W, Kable R, Haley K, Ameh EA. History and development of trauma registry: lessons from developed to developing countries. World J Emerg Surg 2006;1(December (1)):32.

[7] Baker SP, o'Neill B, Haddon [72 TD\$DIFF][63 TD\$DIFF]Jr W, Long WB. The injury severity score: a method for describing patients with multiple injuries and evaluating emergency care. J Trauma Acute Care Surg 1974;14(March (3)):187-96.

[8] Champion HR, Sacco WJ, Copes WS, Gann DS, Gennarelli TA, Flanagan ME. A revision of the trauma score. J Trauma 1989;29(May (5)):623-9.

[9] Boyd CR, Tolson MA, Copes WS. Evaluating trauma care: the TRISS method Trauma Score and the Injury Severity Score. JTrauma 1987:27(April (4)):370-8.

[10] Osler T, Baker SP, Long W. A modification of the injury severity score that both improves accuracy and simplifies scoring. J Trauma Acute Care Surg 1997;43 (December (6)):922-6.

[11] Osler T, Rutledge R, Deis J, Bedrick E. ICISS: an international classification of disease-9 based injury severity score. J Trauma Acute Care Surg 1996;41 (September (3)):380-8.

[12] Champion HR, Copes WS, Sacco WJ, Lawnick MM, Bain LW, Gann DS, et al. A new characterization of injury severity. J Trauma 1990;30(May (5)):539-45.

[13] Kobusingye OC, Lett RR. Hospital-based trauma registries in Uganda. J Trauma Acute Care Surg 2000;48(March (3)):498-502.

[14] Haac B, Varela C, Geyer A, Cairns B, Charles A. The utility of the Kampala trauma score as a triage tool in a sub-Saharan African trauma cohort. World J Surg 2015;39(February (2)):356-62.

[15] Haac BE, Gallaher JR, Mabedi C, Geyer AJ, Charles AG. Task shifting: the use of laypersons for acquisition of vital signs data for clinical decision making in the emergency room following traumatic injury. World J Surg 2017;(July):1-8.

[16] Kelly CA, Upex A, Bateman DN. Comparison of consciousness level assessment in the poisoned patient using the alert/verbal/painful/unresponsive scale and the Glasgow Coma Scale. Ann Emerg Med 2004;44(2):108-13.

[17] Deakin CD, Low JL. Accuracy of the advanced trauma life support guidelines for predicting systolic blood pressure using carotid, femoral, and radial pulses: observational study. BMJ 2000;321(September (7262)):673-4.
[18] Stokes ME, Davis CS, Koch GG. Categorical data analysis using the SAS system. 2nd ed. Cary, NC: Wiley-SAS; 2001. p. 213-6.

[19] Paul P, Pennell ML, Lemeshow S. Standardizing the power of the HosmerLemeshow goodness of fit test in large data sets. Stat Med 2013;32(January (1)):67-80.

[20] Prin M, Guglielminotti J, Mtalimanja O, Li G, Charles A. Emergency-to-Elective surgery ratio: a global Indicator of access to surgical care. World J Surg 2018;42 (July (7)):1971-80.

[21] Haac BE, Gallaher JR, Mabedi C, Geyer AJ, Charles AG. Task shifting: the use of laypersons for acquisition of vital signs data for clinical decision making in the emergency room following traumatic injury. World J Surg 2017;41(December (12)):3066-73

[22] Lavy C, Tindall A, Steinlechner C, Mkandawire N, Chimangeni S. Surgery in Malawi-a national survey of activity in rural and urban hospitals. Ann R Coll Surg Engl 2007;89(October (7)):722-4.

[23] Weeks SR, Juillard CJ, Monono ME, Etoundi GA, Ngamby MK, Hyder AA, et al. Is the Kampala trauma score an effective predictor of mortality in low-resource settings? A comparison of multiple trauma severity scores. World J Surg 2014;38(August (8)):1905-11.

[24] Gardner A, Forson PK, Oduro G, Stewart B, Dike N, Glover P, et al. Diagnostic accuracy of the Kampala trauma score using estimated abbreviated injury scale scores and physician opinion. Injury 2017;48(January (1)):177-83.

[25] Hung YW, He H, Mehmood A, Botchey I, Saidi H, Hyder AA, et al. Exploring injury severity measures and in-hospital mortality: a multi-hospital study in Kenya. Injury 2017:48(October (10)):2112-8.

[26] MacLeod JB, Kobusingye O, Frost C, Lett R. Kampala Trauma Score (KTS): is it a new triage tool? East Cent Afr J Surg 2007;12(1):74-82.

[27] Clarkson CA, Clarkson C, Rubiano AM, Borgaonkar M. A comparison of the Kampala Trauma Score with the revised Trauma Score in a cohort of Colombian trauma patients. Panam J Trauma Crit Care Emerg Surg 2012;1(December (3)):146-9, doi:http://dx.doi.org/10.5005/jp-journals-10030-1032.

[28] Roy N, Gerdin M, Schneider E, Veetil DK, Khajanchi M, Kumar V, et al. Validation of international trauma scoring systems in urban trauma centres in India. Injury 2016;47(November (11)):2459-64.

[29] Samuel JC, Akinkuotu A, Villaveces A, Charles AG, Lee CN, Hoffman IF, et al. Epidemiology of injuries at a tertiary care center in Malawi. World J Surg 2009;33(September (9)):1836-41.

[30] Gallaher JR, Haac BE, Geyer AJ, Mabedi C, Cairns BA, Charles AG. Injury characteristics and outcomes in elderly trauma patients in Sub-Saharan Africa. World J Surg 2016;40(November (11)):2650-7.

[31] Laytin AD, Dicker RA, Gerdin M, Roy N, Sarang B, Kumar V, et al. Comparing traditional and novel injury scoring systems in a US level-I trauma center: an opportunity for improved injury surveillance in low-and middle-income countries. J Surg Res 2017;215(July):60-6.

[32] Weeks SR, Stevens KA, Haider AH, Efron DT, Haut ER, MacKenzie EJ, et al. A modified Kampala trauma score (KTS) effectively predicts mortality in trauma patients. Injury 2016;47(January (1)):125-9.

[33] Akay S, Ozturk AM, Akay H. Comparison of modified Kampala trauma score with trauma mortality prediction model and trauma-injury severity score: a National Trauma Data Bank Study. Am J Emerg Med 2017;35(August (8)):1056-9.

[34] Morris JA, Auerbach PS, Marshall GA, Bluth RF, Johnson LG, Trunkey DD. The trauma score as a triage tool in the prehospital setting. JAMA 1986;256 (September (10)):1319-25.

[35] Koehler JJ, Baer LJ, Malafa SA, Meindertsma MS, Navitskas NR, Huizenga JE. Prehospital Index: a scoring system for field triage of trauma victims. Ann Emerg Med 1986;15(February (2)):178-82.

[36] Gottschalk SB, Wood D, DeVries S, Wallis LA, Bruijns S. The cape triage score: a new triage system South Africa. Proposal from the cape triage group. Emerg Med J 2006;23(February (2)):149-53.

[37] Laytin AD, Kumar V, Juillard CJ, Sarang B, Lashoher A, Roy N, et al. Choice of injury scoring system in low-and middle-income countries: lessons from Mumbai. Injury 2015;46(December (12)):2491-7. 